\title{
¿QUÉ DEBE SABER UN MÉDICO DE PSICOLOGÍA?
}

\author{
Abilio Reig-Ferrer \\ Departamento de Psicologia de la Salud \\ Universidad de Alicante
}

\section{RESUMEN}

La medicina y la psicologia comparten una misma meta: el bienestar de las personas. En esta línea, tanto el médico como el psicólogo son dos tipos de activistas sociales que persiguen ese idéntico fin a través de su actividad profesional sobre la salud, desde la medicina, o sobre el comportamiento de interés, desde la psicología. El estudio de la salud y del comportamiento, como principales focos de atención profesional, se sustenta en determinados modelos de investigación y práctica. El modelo habitual de formación de médicos es el denominado modelo biomédico. Desde la psicología, se ha abanderado una perspectiva biopsicosocial para dar razón de la interacción e integración de los procesos biológicos, psicológicos y sociales tanto en la salud como en la enfermedad. En este artículo queremos destacar la importancia que para la formación, investigación y práctica de la medicina y de sus profesionales tendría la asunción de una perspectiva biopsicosocial desde un modelo de salud centrado en la persona y su contexto.

Palabras clave: MEDICINA; PSICOLOGIA; MODELO BIOPSICOSOCIAL; MODELO BIOMEDICO; MODELO DE SALUD CENTRADO EN LA PERSONA $Y$ SU CONTEXTO. 


\section{SUMMARY}

Medicine and psychology share the same aim: the wellbeing of individuals. In this purpose, physicians and psychologists play the role of social activists who pursue their common goal through their respective professional activities on health, medicine's realm, or behaviour, psychology's domain. The study of health and behaviour, as main sources of professional concem, is based in certain models of education, research and practice. In medicine, the usual model of education is the biomedical model of disease. In psychology, to account for the interaction and integration of biological, psychological and social processes in health and illness, the biopsychosocial perspective has been the brenchmark. In this article, we want to show that it would be beneficial for medicine, its education, research and practice, and its professionals, to incorporate the biopsychosocial perspective within a health model centered in the person and the person's context.

Key words: MEDICINE; PSYCHOLOGY; BIOPSYCHOSOCIAL MODEL; BIOMEDICAL MODEL; HEALTH MODEL CENTERED IN THE PERSON AND HIS CONTEXT.

\section{INTRODUCCIÓN}

Al aceptar la invitación del profesor Vicente Pelechano de reflexionar acerca de qué debe saber un médico de psicología recordé aquellas palabras con las que Teresa de Jesús comienza su tratado de las Moradas: "Pocas cosas que me ha mandado la obediencia se me han hecho tan dificultosas como escribir ahora cosas de oración" (Teresa de Jesús, 1577/1940: 323). Porque, por de pronto, esa propuesta me parece, como Josué ante las murallas de Jericó, para darle vueltas y vueltas. Entre tanta revuelta, me decido, como indigente y menesteroso de conocimientos que soy, por tomar más nota de la de Ávila (entiéndase por oportunismo, no necesariamente por secuacidad teresiana) y recuperar cierto sosiego con aquello de: " [...] que a quien tan poco sabe como yo, forzado habrá de decir muchas cosas superfluas y aun desatinadas, para decir alguna que 
acierte. Es menester tenga paciencia quien lo leyere, pues yo la tengo para escribir lo que no sé, que cierto algunas veces tomo el papel como una cosa boba, que ni sé qué decir, ni cómo comenzar" (pág. 328).

Entrando a machetazos, se puede decir que la medicina es una disciplina en permanente construcción. A semejanza del sujeto de su objeto de estudio se vale de un conocimiento, entreverado de arte y ciencia, vulnerable y caducable. Un conocimiento dirigido a vérselas y arreglárselas con un hombre que es, según la famosa máxima caldea, "un animal de naturaleza variada, multiforme y tornadiza" (Pico della Mirandola, 1988; 55).

La historia de la medicina española parece, en gran medida, una historieta de propuestas para resolver el problema del sufrimiento del hombre. Por sus páginas vemos a un médico azacaneado, en primer lugar, por la salvación del enfermo y de su alma; ocupado, posteriormente, por la vida y curación del paciente; y, ya en la actualidad, intentando centrarse más en la sanación y la calidad de vida de la persona. Aquel primer médico hace cosas pensando más en el otro mundo; en advertir, como su primera obligación, al enfermo grave de la necesidad de la confesión, de facilitar no tanto su sanación como su salvación, ya que como se dice en las Partidas alfonsianas "las almas son mejores que los cuerpos, e más preciadas". Esa era la meta médica de santidad de vida, asegurar una dicha de vida en el más allá. No sorprende, pues, que una ordenanza promulgada en tiempos de los Reyes Católicos, según nos descubre Lain Entralgo (1983), estableciera que el médico que hiciese dos visitas a un enfermo sin indicarle la obligación de confesarse sería multado con diez mil maravedís.

La preocupación médica por una vida sublunar de más años sólo pudo ser posible con la experimentación e identificación de principios farmacológicos activos, policía sanitaria, y un poco de aliño en el estilo de vida personal. Haciendo un repaso histórico a los determinantes del crecimiento de la población de Inglaterra y Gales, McKeown (1976) notó que a partir del siglo XVIII las tasas de mortalidad comenzaron a descender y que esta tendencia parecía ser el resultado de una reducción en el número de muertes causadas por infecciones ligadas a factores nutricionales y ambientales aso- 
ciados a la pobreza. Las conclusiones textuales de McKeown fueron que: "por orden de importancia, los factores que en mayor medida han contribuido a la mejora de la salud en Inglaterra y Gales han sido la limitación del tamaño familiar (un cambio comportamental), una mejor nutrición, un ambiente físico más saludable, y la adopción de medidas preventivas y terapéuticas específicas". Con una contundencia similar, añade el Dr. John Ashton: "Si examinamos el patrón actual de enfermedades predominantemente no-infecciosas (cardiacas, ictus, cáncer, accidentes y suicidio) podemos llegar a la conclusión de que en el futuro las cosas seguirán igual, es decir, que el papel de la medicina continuará siendo de alivio del sufrimiento producido por algunas enfermedades y de aplicación de medidas específicas de prevención desde la clínica. Las grandes mejoras en salud provenientes de la reducción de las muertes prematuras y la promoción del bienestar se obtendrán primariamente de actuaciones fuera del sector médico" (Ashton, 1987: 11).

Por ello, sostenemos aquí que la medicina precisa poca psicología mientras mantenga, y se atenga a, un modelo biomédico de atención sanitaria. Únicamente con un cambio hacia un modelo de salud centrado en la persona y su contexto, la medicina precisa de una "psicología de cabecera", por lo que demandará no sólo más formación en psicología sino que necesitará de la actuación de los psicólogos como profesionales de la salud. Apuntando en esta línea, escribimos las páginas que siguen.

\section{LA PERSPECTIVA BIOMÉDICA DE LA ENFERMEDAD}

Los progresos de la medicina actual tienen mucho que ver con la decisión que en el año 1914 tomó la Fundación Rockefeller de crear una serie de instituciones educativas para la formación de profesionales de la salud. Para ello, propuso a diversas y prestigiosas universidades la presentación de sus propuestas formativas. La Universidad de Harvard, por ejemplo, presentó un modelo de formación en salud de naturaleza ambientalista. La Universidad de Columbia en Nueva York defendió, por su parte, un modelo sociopolítico de formación profesional. Por último, la Universidad Johns Hopkins 
de Baltimore presentó una propuesta biomédica de formación médica que fue por la que finalmente se decidió la Fundación y fue la que inspiro el informe Medical Education in the United States and Canada de Abraham Flexner (White, 1991). En este informe se apostaba por una formación médica basada en el conocimiento experimental a partir de la investigación realizada en enfermedades infecciosas, y por una separación entre lo biológico y lo social, lo curativo y lo preventivo, lo individual y lo colectivo, lo privado y lo público.

Desde entonces, la medicina ha venido actuando sobre la salud desde el modelo biomédico de enfermedad, una perspectiva positivista, reduccionista, dualista y somática en la que se han formado los profesionales sanitarios hasta prácticamente la actualidad. Esto no tendría más trascendencia si no fuera porque los modelos profesionales tienen mucho que ver en cómo entender la salud y la enfermedad, qué tipo de atención prestar, a quién y con qué tipo de relación, desde dónde, o qué papel juegan los otros profesionales de la salud, los familiares y los allegados. Este modelo de trabajo centrado en la enfermedad atiende a determinadas variables de interés a la par que se desentiende de otras. Esta exclusión no significa necesariamente que estas variables no sean importantes; únicamente que no se consideran por no ser de la propia competencia o responsabilidad del médico o por no tener evidencia sólida de su importancia. Identificar la patología (disease) y tratarla ("encontrar y arreglar") es la divisa de este modelo que ha resultado tener un gran éxito en la atención a la enfermedad aguda, sobre todo, la de tipo infeccioso. Se asume que cualquier dolencia o enfermedad (illness) tiene una única causa subyacente (la patología o disease); identificada ésta se inicia necesariamente el tratamiento, por el que se elimina aquella causa y se remite al enfermo de nuevo a la salud. Secunda el modelo biomédico un pensamiento linear a través del cual la patología se puede descomponer en un conglomerado de partes componentes (reduccionismo), susceptibles de análisis, y con la creencia de que la causa y efecto se puede describir mediante una relación. Se insiste, además, en que la enfermedad física tiene una base biológica que sólo puede ser abordada por un técnico experto, el médico. 
El modelo biomédico de enfermedad es el paradigma dominante de atención e investigación sanitarias en la actualidad. En este modelo centrado en la patología, el papel del enfermo es de mero receptor pasivo y paciente del tratamiento; su sistema de creencias, obsoleto y su opinión, cuestionable. El paciente se limita a recibir lo que el médico hace, o coopera y obedece a lo que el profesional le comunica que debe hacer. La salud aquí es la meta última, un fin en sí mismo, por lo que el impacto de la intervención sanitaria en la faceta sociopersonal del paciente y de su familia es irrelevante. Construir salud, desde esta perspectiva biomédica, significa edificar centros sanitarios, básicamente, centros con internamiento (hospitales, por ejemplo) y centros proveedores de asistencia sanitaria sin intemamiento (centros de atención primaria, de salud, polivalentes, de transfusión, consultas médicas, entre otros). Desde aquí parte la oferta asistencial integrada en una serie de servicios o unidades asistenciales (B.O.E. 2003a). Hay fenómenos psicológicos, sin embargo, de agrado biomédico: los efectos placebo y nocebo (sus componentes, mecanismos de acción, variables moduladoras, utilidad) o la adhesión terapéutica, por ejemplo; y aspectos de cierto desagrado: el incumplimiento inteligente, el paciente experto, o la atención a la familia del enfermo. La futilidad en definitiva del conocimiento psicológico que precisa un médico de vertebración biomédica queda palmariamente reflejado en la escasa demanda de formación psicológica del médico. Según el estudio de Waldstein, Neumann, Drossman y Novack (2001), la formación en contenidos de psicología de la salud o de medicina psicosomática es inferior a cuatro créditos en prácticamente la mitad de todas de las facultades de medicina de los Estados Unidos. Un hallazgo similar se ha comentado para Cataluña (Demarbre Zboril, 2001), y esta situación podría ser generalizada para el conjunto de España. Este desinterés por lo psicológico, al menos, se palpa inclusive en la propia psiquiatría. En este sentido, Álvarez Pelaez (2004) revisa la historia reciente de la psiquiatria española y apunta la tesis de la existencia de una aproximación a la etiología y a la terapéutica de las alteraciones mentales basada en la consideración de que esas alteraciones tenían, de forma casi absoluta, una base biológica. 
Pero hay que decir también que el modelo biomédico funciona adecuadamente en el sistema y política de salud generados a su alrededor. La sociedad y el paciente están satisfechos. En una de las últimas encuestas de salud (año 2002), el $82,2 \%$ de la población expresa estar satisfecha o muy satisfecha con los servicios sanitarios utilizados durante el último año, un $80 \%$ de la población refiere estar también satisfecho o muy satisfecho respecto a la última visita a un profesional sanitario en las dos semanas anteriores a la entrevista, y otro porcentaje similar de satisfacción se declara entre las personas que han sido hospitalizadas en el último año (Brugulat, Séculi, Medina et al., 2003).

La evidencia científica disponible en la actualidad rechaza, sin embargo, los supuestos nucleares del modelo biomédico. Este modelo atiende sólo parcialmente los requerimientos de la problemática crónica de salud, el problema de salud dominante en la actualidad, y no entiende la complejidad subyacente a la misma (Holman, 2004). Muchos pacientes, por ejemplo, se presentan con sintomatologia que no se puede atribuir a una causa subyacente o a patología (Wade y Halligan, 2004). La somatización, la tendencia a experimentar y comunicar malestar psicológico en forma de síntomas físicos, explica una buena proporción de la atención sanitaria. Se da el hecho de que en algunos casos, la identificación y análisis del problema de salud no produce mejoría ni en la calidad de vida ni en la expectativa de vida, y circunstancias además en las que la enfermedad, si no es detectada, tampoco tiene un impacto significativo ni en la cartidad ni en la calidad de vida (Kaplan, 2003).

El modelo biomédico de formación en salud ha posibilitado de manera extraordinaria la especialización de sus profesionales. Nadie discute que la especialización médica, ligada también a la progresiva complejidad de la práctica clínica, ha sido una razón muy poderosa del impresionante progreso del conocimiento en la medicina actual. Pero junto a este reconocido honor se apela al horror de su escasa preocupación por una auténtica atención integral de la persona enferma. La práctica clínica especializada del siglo XXI es un ejercicio de la medicina efectivo pero también potencialmente peligroso , con presencia de errores médicos, o los denominados efectos adversos evitables (lesiones o complicaciones que dan lugar a in- 
capacidad, muerte o prolongación de la estancia hospitalaria y que están causados por el sistema sanitario más que por la enfermedad del paciente) (Aranaz, Aibar, Gea, y León, 2004).

En este contexto, el conocimiento psicológico que precisa un médico de formación y práctica biomédica es casi anecdótico. Con buenas pruebas biológicas, la información del paciente es poco relevante. Es el laboratorio el lugar donde se identifica la mayor parte de la información requerida para el diagnóstico y tratamiento. El sistema de creencias del enfermo no aporta nada de interés. En todo caso, son los factores ambientales el foco de atención, no el ambiente social o el mundo personal del individuo. Hay una única manera de estar enfermo y un único modo de rol de enfermo, de funcionar y ser un buen paciente. Se palpa, pues, una gran homogeneidad en el camino del enfermar y de la enfermedad. Frente a este estado de cosas, Pelechano, por ejemplo, desenmascara la carencia de modelos científicos en salud que reconozcan la variabilidad de formas de estar enfermo y sano: "Uno de los desafíos que existen en nuestra sociedad es, junto al reconocimiento de la diferenciación humana, el de la aceptación de modos, estilos de vida y formas de vivir que representan modelos distintos de estar en el mundo y de estar "sanos". [...] lo que queremos decir es que al igual que existen muchas maneras distintas de convivir y afrontar una enfermedad crónica, asimismo existen, muy posiblemente, muchas maneras distintas de "estar relativamente sano" o de padecer una cierta enfermedad que no posee necesariamente un pronóstico mortal inmediato $y$, durante ese periodo de mantenerse vivo, operativo $y$ activo, existen una diversidad de posibilidades de acción que tienden a ser ignoradas y pensamos que bien merecerían estudios especiales al respecto" (Pelechano, 1996b: 370-371).

No sólo los programas de formación sanitaria, sino el diseño de hospitales y centros de salud, la creación de especialidades médicas, la industria sanitaria y farmacéutica, o las propias políticas de salud emanan del carácter hegemónico del modelo biomédico de atención a la enfermedad.

La medicina o práctica basada en la evidencia, en su deseo por conocer racionalmente la enfermedad, integrando la mejor evidencia científica disponible junto con el más alto grado de pericia clínica, 
parece dar la espalda de nuevo a la persona al no incorporar satisfactoriamente los valores del paciente (Lockwood, 2004). Cualquier profesional de la salud tiene la obligación de utilizar esta evidencia de una manera crítica de modo que se desarrolle una respuesta para cada paciente, para cada problema nuevo o específico y se valore el resultado. La nueva genética, en esta línea, podría suponer una nueva ola de reduccionismo en las ciencias de la salud. Así, el denominado Proyecto Genoma puede influir en la opinión del público restando importancia a los factores personales y medioambientales del enfermar y de la enfermedad, generando de nuevo una nueva era en la cultura de la salud: la era de la higiene interior (Davo y Álvarez-Dardet, 2003).

No es lugar aquí de intentar mostrar todas y cada una de las disfunciones y patologías del modelo médico de enfermedad sino de hacer más conspicuo que nos encontramos con un sistema sanitario poco propicio para una medicina centrada en la persona. Una medicina azorada todavía más en curar que en sanar, en ganar años a la vida a costa de pasarlos en peor salud. Sanar, recuérdese, no implica necesariamente curar, sino que apela a ayudar a la persona para que consiga un modo de vida compatible con sus aspiraciones; reestablecer, en definitiva, su libertad para tomar decisiones, inclusive en la presencia de situaciones permanentes de enfermedad (Dubos, 1978).

Se escuchan voces, aunque no necesariamente aquellas estruendosas que profería el bárbaro Corsicurbo del que nos habla el autor de Los trabajos de Persiles y Segismunda, acerca de la necesidad de "transmitir a las generaciones médicas futuras que la poderosa medicina actual basada en la evidencia debería amasarse con una buena dosis de medicina basada en la afectividad" (Jovell, 1999; Marañón Cabello, 2004); de la necesidad de "cambiar el paradigma de actuación y empezar a valorar los resultados que obramos con medidas centradas en los pacientes. Ir a buscar el resultado donde realmente se produce y en la forma que es importante para un individuo" (Lizán, 2004: 144).

En una entrevista reciente, el doctor Ciril Rozman, unos de los autores del célebre tratado de consulta médica Farreras-Rozman: Medicina Interna, se lamentaba del impacto que el excesivo número 
de especialistas tiene sobre una atención sanitaria fragmentada: “L'essència de la medicina interna és, en primer lloc, el contacte amb el malalt, peró si el primer contacte és dolent, l'eficacia de l'acte mèdic s'ha acabat. Després hi ha l'exploració física, que no es pot suplir mai mitjançant proves. Per aixo, un especialista no es pot limitar a entendre només de la seva especialitat, sinó que ha d'escoltar totes les queixes del malalt. I és que la tragèdia de l'assistència fragmentada ha arribat a l'extrem que ja no la dirigeixen els metges, sinó els malalts. [...] Vull dir que són els malalts el qui trien els seus especialistes i després els expliquen el que ells consideren que és de la incumbència d'aquests professionals, i callen el que no ho és tant. Peró de vegades el que callen és, justament, la clau del diagnóstic" $Y$ más adelante. "Actualment, es tendeix molt a parlar d'una medicina basada en l'evidència, pèro jo, en cavi, defenso una medicina basada en la incertesa. Els metges hem d'exercir sempre amb un cert grau d'incertesa, sense que aixo ens impedeixi escollir què és el millor per al malalt" (Alcaraz, 2005) ${ }^{1}$.

En su práctica diaria el médico tiene que tomar decisiones sin prácticamente más apoyo que su capacidad de juzgar y razonar para decidir lo que es bueno para el paciente. La norma de "más es mejor" preside el logro de un mayor nivel de vida pero no, necesariamente, de un mayor respecto al enfermo o de una mayor calidad de vida. Las decisiones médicas nunca son ciertas pero son opiniones sopesadas y de calidad que se apoyan en la mejor evidencia disponible. No obstante, el mejor criterio en la toma de decisiones es,

1. "La esencia de la medicina interna es, de entrada, el contacto con el enfermo; pero si este primer contacto es malo, se acaba la eficacia del acto médico. Está, después, la exploración física, que no se puede nunca sustituir por pruebas. Por ello, un especialista no se puede limitar a saber únicamente de su propia especialidad sino que debe escuchar todas las demandas del enfermo. $Y$ es que la tragedia de la asistencia fragmentada ha llegado al punto de que ya no la controlan los médicos sino los enfermos. [...] Quiero decir que son los enfermos los que escogen a los especialistas y después les explican lo que creen que es de la incumbencia de estos profesionales, callando lo que no lo es tanto. Pero, a veces, lo que callan es justamente la clave del diagnóstico [...] Se tiende mucho en la actualidad a hablar de una medicina basada en la evidencia pero yo, en cambio, defiendo una medicina basada en la incertidumbre. Los médicos hemos de ejercer siempre con ciento grado de incertidumbre, sin que ello impida elegir to que es mejor para el enfermo". 
insistimos una vez más, el deseo o prioridad de la persona afectada. La responsabilidad del médico será siempre la de señalar e intentar modificar aquellos comportamientos que pudieran acortar o disminuir la calidad de vida de sus pacientes, pero éstos deberían de gozar siempre de la más absoluta responsabilidad para tomar decisiones libres en lo que atañe a su vida y a su salud. Se le suponen, por tanto, al médico toda una serie de hábitos de comportamiento bueno: la voluntad de hacer el bien (benevolencia), respeto a la persona, prudencia (el arte de elegir bien, distinción clara entre lo comprobado, que suelo ser mucho, y lo dudoso, que suele ser mucho más), confianza, fidelidad (es decir, atenimiento a los datos y a las pruebas), honestidad, justicia y coraje. Pero no basta con querer obrar bien, hay que aprenderlo y saberlo hacer, aprender un hábito de prudencia en el comportamiento médico, sustentado en la incertidumbre de la complejidad del proceso de salud (Sanz Ortiz, 2002).

La formación que están recibiendo los médicos de familia, se plantean Turabián Fernández y Pérez Franco (2004), no está respondiendo al elemento central que es identificar la experiencia relacional del paciente y considerarla globalmente: "Los estudiantes y residentes saben que los elementos psicosociales que caracterizan la vida de una persona tienen un impacto en su enfermedad, y que la persona es el sujeto y objeto de la asistencia médica, pero son incapaces de ponerlo en práctica con la intensidad necesaria para sustituir a la visión tecnológica y la medicina centrada en la patología. [...] La tecnología produce una fascinación que favorece su perpetuación. Así, el médico, ante un paciente que sufre, no habla sobre su sufrimiento o sobre qué puede hacer por el enfermo...; no, el médico lee los resultados de las analíticas y de las radiografías, lo que es muchas veces irrelevante para el complejo problema más inmediato del paciente, pero que da la apariencia de resultados inmediatos y sencillos" (pág. 507). No deja de sorprender, en este contexto, que el tiempo de escucha activa por parte del médico sea mínimo. Seguí Díaz et al. (2004) han encontrado que el tiempo medio por encuentro médico es de 9,1 minutos, y el tiempo medio por problema atendido es de 7,4 , con un recorrido entre los 76 segundos y los 25 minutos. 
La psicología apuesta por una medicina centrada en la persona y por una relación con los médicos basada en la cooperación y colaboración. El diálogo entre ambas disciplinas no ha sido fácil, y la falta de cierta armonía de encuentro, como señala Pérez Álvarez, ha caracterizado buena parte de su relación: "Las relaciones entre la Medicina y la Psicología han estado desarmonizadas, unas veces porque los médicos, debido a una formación internalista autosuficiente, no han visto interés efectivo en las circunstancias psicológicas del paciente, y otras porque la aportación de los psicólogos a un tal interés resultaba, a menudo, inútil, aunque, quizá, curiosa, y, en ocasiones, ridícula" (Pérez Álvarez, 1990: 11).

\section{¿TIENE NECESIDAD LA MEDICINA DE MÁS PSICOLOGÍA?}

En mi experiencia como profesor vinculado a la formación de médicos (desde 1982 hasta 1997) y de personal de enfermeria (desde 1997 hasta la actualidad) he sido testigo del grado de enraizamiento de la psicología en el conocimiento y práctica de las ciencias de la salud en España. La psicología, y sus respectivas áreas de conocimiento, está presente académicamente en la formación de profesionales sanitarios a través de diversas asignaturas, algunas de ellas troncales como "Bases psicológicas de los estados de salud y enfermedad" (facultades de medicina) o "Ciencias psicosociales aplicadas a la salud" (escuelas universitarias de enfermería), que permiten proporcionar conocimientos básicos, destrezas y actitudes acerca de las relaciones entre el comportamiento y la salud; de las teorías, modelos y objetivos de la atención sanitaria; del estrés y de su afrontamiento; de la psicobiología de los procesos de enfermar y enfermedad; de la psicología de la sintomatología física; de la agenda del paciente: sus creencias e intenciones; del impacto psicológico de la enfermedades, del proceso de morir y del duelo; la psicologia de las decisiones del profesional sanitario, la comunicación clínica y la adhesión al tratamiento; de la importancia de dotar de poder (empowerment) al paciente con los elementos de información, elección y control; de los aspectos psicológicos de la hospitalización y de las intervenciones quirúrgicas; de los tratamien- 
tos psicológicos disponibles basados en la evidencia para el tratamiento de diversa sintomatología y de una amplia gama de problemas de salud; o de la importancia de utilizar la perspectiva del paciente para valorar la atención sanitaria. Algunos manuales recientes inciden precisamente en la aportación de algunos de estos conocimientos de interés para la formación de cualquier profesional de la salud (entre otros, Gil Roales-Nieto, 2004; Jiménez Jiménez, 2001; León Rubio, Medina Anzano, Barriga Jiménez, Ballesteros Regaña y Herrera Sánchez, 2004; Nieto Munuera, Abad Mateo, Esteban Albert y Tejerina Arreal, 2004).

La psicologia está apostando con firmeza por ser considerada como una disciplina de carácter sanitario. La todopoderosa American Psychological Association modificó en el año 2001 su "mission statement" (declaración de principios) para incorporar explícitamente el término "salud" con la aprobación de más del $95 \%$ de todos sus miembros. Desde entonces, la misión de la psicología es " [...] to advance psychology as a science and a profession, and as a means of promoting health and human welfare" (Rozensky, Johnson, Goodheart, y Hammond, 2003).

En España, durante estos últimos años se está haciendo un esfuerzo considerable para el reconocimiento de la psicologia como profesión sanitaria, ya que en la actualidad, por ley, tan sólo tienen la consideración de profesionales sanitarios los psicólogos que ostenten la titulación de Especialista en Psicología Clínica. La Ley 44/2003, de 21 de noviembre, de ordenación de las profesiones sanitarias (LOPS) (B.O.E., 2003b) ha supuesto, pese a sus limitaciones, carencias y ambigüedades, un paso inicial en la consideración del psicólogo como profesional sanitario a través del título de psicólogo especialista en psicología clínica. A este nuevo profesional sanitario se le adjudica una determinada actividad sanitaria de psicología clínica. El Real Decreto 1277/2003, de 10 de octubre, por el que se establecen las bases generales sobre autorización, servicios y establecimientos sanitarios (B.O.E., 2003a) define "actividad sanitaria" como el "conjunto de acciones de promoción, prevención, diagnóstico, tratamiento o rehabilitación, dirigidas a fomentar, restaurar o mejorar la salud de las personas realizadas por profesionales sanitarios" (pág. 37894). Se establece, entre la oferta asistencial, 
servicios o unidades asistenciales de "Psicología clínica", y entendiendo éstas como una "unidad asistencial en la que un psicólogo especialista en Psicología clínica, dentro del campo de su titulación, es responsable de realizar diagnósticos, evaluaciones y tratamientos de carácter psicológico de aquellos fenómenos psicológicos, conductuales y relacionales que inciden en la salud de los seres humanos" (pág. 37901). Incomprensiblemente, o por descuido intencionado, no se alude a la competencia del psicólogo para el diagnóstico, la evaluación, el tratamiento, y la rehabilitación de los trastornos mentales y emocionales.

Pero la actuación de la psicología y de sus profesionales en el campo de la salud no se puede cerrar a una única área de actuación. El psicólogo es experto en toda una serie de repertorios comportamentales relevantes que abarcan toda la atención sanitaria: comportamientos que mantienen la salud y el bienestar, comportamientos que previenen la enfermedad y el malestar, comportamientos de riesgo para la salud, comportamientos que constituyen recursos cuando se ha detectado una enfermedad, y comportamientos que previenen la cronicidad, la discapacidad y la invalidez. Por ello, la atención psicológica y comportamental en otras unidades asistenciales es prioritaria: Planificación familiar, Interrupción voluntaria del embarazo, Tratamiento del dolor, rehabilitación, Terapia ocupacional, Cuidados paliativos, Psiquiatría, Atención sanitaria a drogodependientes, Medicina preventiva, Medicina de la educación física y del deporte, Extracción de órganos, etcétera. La presencia de psicólogos tanto en atención especializada como en atención primaria y los servicios de Unidades de conductas adictivas, planificación familiar, etc., están siendo un recurso de gran interés para la consolidación de la psicología clínica y de la salud en nuestro sistema de salud pero disponen de una buena sedimentación en otros países (Bray, 2004; Hong y Leventhal, 2004; Johnson, 2004; King, 2004; Kush, 2001; Tovian, Rozensky y Sweet, 2003).

Pero, ¿qué opinión tienen nuestros médicos de la psicología? Por los resultados que me adelanta Buela-Casal de una reciente encuesta a unos 600 profesores de medicina y de psicología sobre la opinión que les merece la psicología como profesión sanitaria, los médicos tienen una imagen del carácter sanitario de la psicología 
muy similar a la que refieren los propios psicólogos (Buela-Casal, Gil Roales-Nieto, Sierra, Bermúdez, Agudelo, Breton y Teva, 2005).

La psicología está cada vez más presente en las revistas médicas especializadas. Por poner un ejemplo de alcance general, la revista Jano, Medicina y Humanidades, de éxito continuado, reconocimiento, aceptación y amplísima distribución entre profesionales de la medicina, ofrece una ventana a la que asomarse la psicología con la denominación de "Psicología de la salud". Médicos de atención primaria, psiquiatras, y también psicólogos presentan artículos de opinión sobre temas de actualidad sanitaria. La propia denominación de psicología de la salud y la publicación de opiniones, sobre todo, de profesionales de la medicina resulta ser un aspecto atractivo adicional para su consulta. A pesar de la carencia de un estudio detallado en el ámbito español, Suls y Rothman (2004) han evaluado el grado de incorporación de la psicología, desde una perspectiva comportamental, en el campo de la medicina. Para ello, han tabulado la frecuencia de aparición de la palabra "comportamiento" (behavior) desde 1974 hasta 2001 en cuatro de la principales revistas médicas: New England Journal of Medicine, Lancet, Joumal of the American Medical Association (JAMA), y Annals of Intemal Medicine. En los primeros años, la palabra comportamiento apareció en los títulos o resúmenes de 61 artículos frente a las algo más de100 de los últimos años analizados (casi el doble de aparición). Teniendo en cuenta el número de artículos totales publicados en las cuatro revistas, el término "behavior" se mencionó un $0,25 \%$ en los primeros años revisados (1974-1977) frente a un $0,41 \%$ al comienzo de los años 90. A pesar del incremento, se puede concluir que la psicología todavía tiene una mínima presencia, al menos utilizando este indicador, en las revistas de medicina.

Es verdad que la entrada de la psicología en la arena sanitaria es progresiva y continuada aunque lenta. Cada vez trabajan más psicólogos en centros de formación para profesionales sanitarios y en centros sanitarios (Hong y Leventhal, 2004), pero todavía queda mucho trabajo por hacer. Los psicólogos hemos estado trabajando casi como traperos cientificos, buscando datos y cargando con un gran fardo de resultados, pero como meros colectores de hechos nuevos. Se ha pretendido, en este sentido, más encontrar que pensar; 
hemos aspirado más a templar que a contemplar. Por esa urgencia de demostrar, hemos dado la espalda al alarife, al arquitecto del conocimiento. Ahora precisamos de alarifes psicológicos. Se ha amasado una considerable evidencia sobre la relevancia del conocimiento psícológico y sus aplicaciones al marco de la salud y de la enfermedad a pesar de que todavía precisemos de conocimiento en mayor medida que explicación en muchas áreas de la psicología de la salud. De fenómenos psicológicos clásicos como el efecto placebo, del que conocemos algunas claves de su proceso y resultados (Ferreres, Baños, y Farré, 2004; Pérez Álvarez, 1990; Sierra, Carrasco, y Buela-Casal, 1995; Sierra, Luna-Villegas, Buela-Casal, y Femández-Guardiola, 1996), apenas conocemos la frecuencia, actitudes y circunstancias de su uso en el contexto de la práctica clínica diaria (Nitzan y Lichtenberg, 2004). Muy poco se ha estudiado el comportamiento de desviación positiva (Marsh, Schroeder, Dearden, Sternin \& Sternin, 2004) o lo mucho que los profesionales de la salud podemos aprender de los cuidadores familiares 0 de los propios pacientes para la conformación de nuevos tratamientos y formas de ayuda. No hay duda de la apuesta psicológica por ayudar a una medicina que asuma como propio un modelo de atención de salud en el que el foco no sea exclusivamente combatir la enfermedad sino reconstruir la salud; la necesidad de un mayor énfasis en las variables comportamentales de la mano de las socioambientales; de una causación de la problemática de salud producto de la interacción de los patógenos y de los huéspedes; de la consideración del usuario como agente activo de salud, colaborador en el tratamiento en el proceso de sanación; o de la importancia de su sistema de creencias $y$ de su faceta sociopersonal (Ray, 2004).

La cascada de nuevos resultados que confirman otros previos 0 que apuntan novedades de interés está siendo imparable. No hay prácticamente rì un sólo profesional de la salud que no reconozca la importancia del estilo de vida de las personas como determinante sustantivo de los procesos de salud y enfermedad. Pero el profesional sanitario debe saber asimismo que el comportamiento es la cuestión central en la atención sanitaria (Kaplan, 1990, 1994). Sólo una condición biológica, emocional o mental tiene interés si tiene impacto en la cantidad y calidad de vida. El comportamiento cons- 
tituye el medio de contacto directo e indirecto con agentes patógenos y nocivos a través de la acción, del pensamiento, del afecto o de la intención; a través de nuestro comportamiento modulamos los efectos biológicos de las circunstancias ambientales y sociales hasta su traducción inmunológica; con nuestro comportamiento sufrimos y nos matamos (con los comportamientos de riesgo) o nos salvamos (con las prácticas de salud, de seguridad, de cuidado preventivo, o de evitación de sustancias y contextos peligrosos); a través del comportamiento se potencia cualquier acción diagnóstica y terapéutica, etc. La idea de que un estilo de vida saludable prolonga la vida es un hecho que se confirma en la literatura científica una y otra vez. Inclusive en personas de más de 70 años, la adhesión a una dieta mediterránea, un uso moderado del alcohol, no fumar y realizar algo de ejercicio físico se asocia a una disminución de una tasa de más del $50 \%$ en mortalidad, tanto global como específica (Knoops et al., 2004). Afrontar nuestro desarrollo ontogenético en positivo (presencia de autopercepciones positivas del proceso de envejecimiento frente a los estereotipos negativos de la vejez) se relaciona, en personas mayores, con una supervivencia añadida de 7 años y medio de vida (Levy, Slade, Kunkel \& Kasl, 2002). Un estilo explicativo optimista similar disminuye, también en ancianos, el riesgo de mortalidad por enfermedad coronaria (Kubzansky, Sparrow, Vokonas, \& Kawachi, 2001), mientras que un estilo explicativo pesimista se asocia significativamente con mortalidad prematura (Maruta, Colligan, Malinchoc, \& Offord, 2000). Y con evidencias similares se está escribiendo día a día la psicología de la salud.

Si un profesional competente es el que utiliza los conocimientos científicos, habilidades, actitudes y un "buen juicio" asociados a una profesión para atender adecuadamente las "situaciones" planteadas en su ejercicio, se debe ir más allá de la realización correcta de diagnósticos, pronósticos y prescripciones de tratamientos. Se habla cada vez más de una nueva conciencia de la complejidad (Pelechano, 1996a) para dar cuenta de que la realidad no es simple sino diversa y compleja: "Ni la medicina es simple ni, mucho menos, lo es la psicología. $Y$ cuando algo es explicado con simplicidad parece más obra de traición a la realidad y un canto a la ineficacia que un esfuerzo por generar modelos científicos que sean capaces de generar 
investigación acumulativa y cercana a la realidad biológica y psicoIógica" (Pelechano, 1996a: 340). En este mundo complejo, se debe formar no tan sólo para la competencia (lo que el individuo sabe o es capaz en términos de conocimiento, actitudes y habilidades) sino para la capacitación, entendida como la capacidad que permite adaptarse a los cambios, generar nuevos conocimientos y mejorar continuamente nuestro desempeño y ejercicio profesional. Esta capacitación se aviene bien con el carácter cambiante de las situaciones prácticas, complejas, holísticas, inciertas, inestables y únicas de la práctica clínica.

A pesar de que los modelos conceptuales de formación y práctica en medicina siguen aferrados a la perspectiva biomédica de enfermedad, en enfermería, sin embargo, las teorías y modelos de mayor frecuencia de aparición en las revisiones de la literatura presentan un marcado acento psicológico y a distancia de otros posicionamientos teóricos provenientes de la fisiología, de la sociología o de la antropología (Cabrero y Richart, 2001). Estos autores constatan en el campo de la investigación enfermera una preponderancia de los modelos psicosociales sobre los biológicos, sobre todo en la enfermería europea. Los modelos de formación en enfermería están preparando más adecuadamente a este profesional para un mundo de la salud complejo, necesitado de colaboración interdisciplinar, centrado en la personas y sus contextos de vida, $y$ ante una problemática sanitaria dominada por las enfermedades crónicas que han reemplazado a la enfermedad aguda como problema de salud dominante.

Las enfermedades crónicas son hoy en día la principal causa de discapacidad y de utilización de los servicios de salud consumiendo el $78 \%$ del gasto sanitario. No menos importante, la enfermedad crónica ha cambiado radicalmente el papel del paciente y el de sus allegados. ¿Están formados los profesionales sanitarios para afrontar esta situación? La evidencia disponible avala que no está formado el médico para este cometido (Holman, 2004). Para añadir otro punto de complejidad en la tarea, el médico tiene que atender a una nueva que quiere hacerse escuchar, la de las asociaciones de pacientes, y que está consiguiendo ser una fuente importante de poder sanitario cada vez mayor. Según se desprende del informe 
El paciente en España. Mapa nacional de las asociaciones de pacientes promovido por Farmaindustria, unos 19 millones de españoles presentan una patología crónica, de los que sólo entre 5 y 7 millones reciben asistencia de forma sistemática y continua. Los trastornos de salud más frecuentes tienen que ver con artrosis y problemas reumáticos (29\%), hipercolesterolemia (14,2\%), enfermedades cardiovasculares (14\%), diabetes e hipoglucemia (14\%). Las enfermedades del aparato circulatorio representan el $12,3 \%$ del total de la morbilidad hospitalaria, seguidas de las patologías del aparato digestivo (11\%) y del respiratorio (10\%). Es preocupante el elevado número de enfermos que no acude a recibir asistencia sistemática, y que incluso no sigue tratamiento alguno. Depresiones, alergias, hipercolesterolemia, e hiperglucemias son las patologías en las que se detecta un mayor número de enfermos inadecuadamente atendidos. En general, los pacientes entrevistados valoran positivamente el sistema asistencial, refieren tener un alto grado de conocimiento sobre su enfermedad (siendo el médico, con gran diferencia, su principal fuente de información), están satisfechos de la eficacia de los tratamientos farmacológicos que reciben y, en el orden de prioridad en relación a su enfermedad, valoran en primer lugar los aspectos científicos y terapéuticos $y$, en segundo lugar, los asistenciales y sociales. A pesar de su satisfacción con la información y trato que reciben, afirman que todavía hay mucho que mejorar en cuestión de tratamientos e investigación (Anónimo, 2004).

Con estos mimbres, un buen cesto que recoja y atienda las necesidades básicas de información, apoyo e intervención técnica ligadas a los valores, intereses y prioridades de la persona es el modelo de salud centrado en la persona y en su contexto.

\section{LA PROPUESTA de UN MODELO de SALUd CENTRADO EN LA PERSONA Y EN SU CONTEXTO}

La medicina moderna está comenzando a apostar por un modelo de salud centrado en la persona con una nueva perspectiva de la salud y de la enfermedad, de la vida y de la muerte, de los agentes de salud, y de los contextos de salud. El modelo centrado en la 
persona no tiene en la actualidad un significado inequivoco en medicina. Unas veces se entiende como filosofía de la medicina; otras como imperativo moral y profesional; mucho más frecuentemente se ha operacionalizado como estilo de comunicación, como método clínico, como tipo de relación terapéutica, o finalmente como indicador de calidad de la atención sanitaria. La naturaleza de la enfermedad es importante, pero no central. Lo radical es centrarse en la persona-en-su-contexto. Contexto en su significado de trabazón histórica, de discurso biográfico, no sólo como ambiente o hábitat en el que la gente vive y trabaja, y que engloba a los familiares o allegados. En realidad, muchos problemas crónicos de salud se viven como problemas familiares. El cáncer, por ejemplo, afecta directamente a toda la estructura familiar y sus miembros sufren conjuntamente con el enfermo en la lucha contra el cáncer. Por ello, los miembros de la familia tienen una considerable influencia tanto en el control de la enfermedad como en la adaptación psicológica del paciente. Los profesionales de la salud deben, en este sentido, incorporar a los familiares en la atención sanitaria. Estudios metaanalíticos recientes aportan evidencia que sugiere que la intervención psicológica temprana llevada a cabo con el paciente de cáncer y su cuidador de referencia puede prevenir en el futuro la evolución psicopatológica de resultados de malestar psicológico en ambos (Hodges, Humphris y Macfarlane, 2005); y demuestran también los efectos positivos de las intervenciones psicológicas tanto sobre los resultados de salud del paciente (depresión, y en algunos casos, mortalidad) como del familiar cuidador (sobrecarga, ansiedad, depresión) cuando se incorpora a ambos en el tratamiento (Martire, Lustig, Schulz, Miller y Helgeson, 2004).

Con este modelo de salud se pretende también disminuir ciertos elementos de insatisfacción que no aparecen en las encuestas al respecto y que tienen una relación directa con el incumplimiento terapéutico. La falta de un trato personalizado, de intímidad y privacidad, de información, el escaso tiempo disponible para la atención individual y familiar, las listas de espera y la falta de continuidad asistencial son los elementos que con mayor frecuencia provocan insatisfacción en los ciudadanos (Brugulat, Séculi, Medina, Juncá, Martínez, Martínez, y Treserras, 2003). También conocemos 
que los pacientes se muestran poco dispuestos a criticar a los profesionales de la salud. Hay estudios que demuestran que el modo con el que la persona ha sido tratada en el pasado por los profesionales de la salud determina la búsqueda de tratamiento en el futuro (Moore, Sickel, Malat, Williams, Jackson, y Adler, 2004). Con modelos de regresión logística, estos autores señalan la importancia que tiene la escucha activa de las preocupaciones del paciente para evitar retrasos e incluso evitación del tratamiento médico o psicológico. Se ha analizado también si una posible fuente de vanabilidad en los aspectos interpersonales de la práctica médica puede ser el género del profesional de la salud ya que se ha especulado que las mujeres médico son más "centradas" en la comunicación con sus pacientes. Hay datos que avalarían esta suposición. Así Delgado y López- Femández (2004) mantienen que las médicas de atención primaria tienen un estilo de comunicación más centrado en lo psicosocial que los médicos, a la vez que las mujeres expresan con frecuencia la preferencia por una médica para consultar problemas de indole psicosocial. Para clarificar esta cuestión, Roter y Hall (2004) han realizado dos revisiones meta-analíticas de la literatura desde 1967 hasta 2001en las bases MEDLINE, AIDSLINE, PsycINFO y BIOETHICS. Sus resultados confirman que las mujeres dedican más tiempo al paciente en la visita médica (dos minutos más de media) y se comprometen en un estilo de comunicación significativamente más centrado en el paciente: conductas más activas de colaboración, habla positiva, relación de ayuda de tipo psicosocial, petición de corresponsabilidad de la propia salud, preguntas de referenciación psicosocial y temática más enfocada a los aspectos afectivos. En esta línea, los pacientes de las médicos hablan durante más tiempo, presentan expresiones más positivas, discuten más información biomédica y psicosocial y demuestran un mayor grado de colaboración que los pacientes de médicos masculinos. El género del médico no se relaciona con la provisión de información biomédica (incluyendo discusión del diagnóstico, pronóstico y tratamiento médicos). Curiosamente, únicamente en los servicios de obstetricia y ginecología aparece un patrón diferencial. Aquí son los médicos varones los que mantienen un estilo relacional más centrado en la afectividad y la esfera psicosocial. En definitiva, cuando la atención 
médica se centra en la persona, y desde una perspectiva biopsicosocial, los resultados positivos en salud y satisfacción aumentan significativamente (Delgado y López- Fernández, 2004).

La base conceptual de este modelo de salud centrado en la persona es la denominada perspectiva biopsicosocial (Engel, 1977). Esta perspectiva asume que los procesos biológicos, psicológicos y sociales están comprometidos interactiva e integradoramente tanto en la salud como en la enfermedad. La teoría general de sistemas del biólogo austriaco L. von Bertalantfy conforma la base de la perspectiva biopsicosocial. Se entiende por sistema un agregado de elementos interrelacionados e interconectados que constituyen un todo. Todos los seres vivientes son equipos organizados de sistemas; estos sistemas de vida son un agregado complejo e interrelacionado de elementos organizados jerárquicamente y compuestos de diferentes niveles, por ejemplo: célula - órgano - sistema de órganos - organismo - comportamiento - grupo - organización - sociedad - sistema supranacional. Cada sistema de nivel superior posee características que emergen sólo a ese nivel y un sistema mostrará distintas facetas según sea la escala de observación. Estos fenómenos emergentes existen a pesar de que no son mero surgir de lo material. Se consideran fenómenos de orden distinto a lo material que deben explicarse, al menos parcialmente, a partir de principios no materiales. De un enfoque analítico se pasa a otro holístico o estructural en el que se incorpora lo individual y lo social, lo conductual y lo vivencial, el cuerpo y la mente.

La psicología es fundamental para la medicina con mirada biopsicosocial, pero ¿está adoptando la medicina una perspectiva biopsicosocial? Suls y Rothman (2004) han llevado a cabo una búsqueda a través de la base Medline de las palabras clave "biopsicosocial" y "biomédico", para valorar el reconocimiento de cada una de estas perspectivas. El periodo de tiempo elegido fue de 27 años, desde 1974 hasta 2001. El término "biomédico" aparece en estos 27 años en un total de 9994 referencias frente a "biopsicosocial" que aparece citado un total de 1094 veces (una proporción de 9 a 1). El término "biopsicosocial" se mencionó en 6 artículos durante el periodo de 1974-1977, año este último en el que Engel (1977) lo introduce formalmente en medicina, mientras que 
en los cuatro últimos años analizados, 1998-2001, esta palabra figuraba en 350 publicaciones; es decir, aumenta por 60. Si se comparan ambos periodos con la utilización del término "biomédico" nos encontramos con casi 500 veces (1974-1977) y con 2700 veces (1998-2001); es decir un aumento por 5. Los autores concluyen que a pesar del aumento progresivo y constante de citación de "biopsicosocial", la perspectiva biomédica permanece dominante en el contexto de las revistas de medicina.

Tomando este marco de referencia, los psicólogos de la salud han abanderado, aunque más con palabras que con hechos, este acercamiento multinivel y multisistémico a la hora de abordar el funcionamiento humano. Diversos estudios y revisiones han demostrado el importante papel de la perspectiva biopsicosocial para valorar la comorbilidad de los problemas de salud tanto físicos como mentales y específicamente en los trastornos ligados al dolor (Gatchel, 2004), - la importancia que los afectos, el estrés y el apoyo social juegan en la progresión y mantenimiento de la patología cardiaca y oncológica (Anderson, 2002; Smith \& Ruiz, 2002). Los modelos cognoscitivoperceptuales de sentido común de la salud y de la enfermedad han aclarado aspectos relevantes acerca de la percepción de síntomas, de la adhesión a las recomendaciones sanitarias o del comportamiento de búsqueda de atención sanitaria (Leventhal, Leventhal \& Cameron, 2001). La función protectora de las creencias positivas como recurso de salud y de calidad de vida ha sido está avalada por una sólida evidencia (Taylor et al., 2000). Cohen (2004) aporta distintos tipos de evidencia en relación a que tres tipos diferentes de variables de relación social (apoyo social, integración social, e interacción negativa) se relacionan estrechamente con resultados en salud. Diversos tratamientos psicológicos han demostrado éxito en tabaquismo (Niaura \& Abrams, 2002), en la reducción del estrés y efectos secundarios asociados a procedimientos médicos (Suls \& Wan, 1989), o en la adaptación satisfactoria a la patología crónica (Anderson, 2002; Blumenthal et al., 2002). Una buena guia de tratamientos psicológicos eficaces para diversos problemas de salud de interés para cualquier profesional sanitario se presenta en los tres volúmenes coordinados por Pérez Álvarez, Fernández Hermida, Fernández Rodríguez y Amigo Vázquez (2003). 
En este modelo, la salud y el comportamiento se consideran recursos personales para una vida de cantidad y calidad. La salud se hace y se vive en los contextos cotidianos de vida de las personas (Reig-Ferrer, Cabrero-García, Ferrer-Cascales, y Richart-Martínez, 2001), por lo que conviene dilucidar qué es estar sano, sentirse sano y parecerlo a los demás. Conocemos, por ejemplo, que los jóvenes españoles identifican lo sano con la buena alimentación y la actividad física (Hernán, Fernández y Ramos, 2004; Reig-Ferrer, CabreroGarcía, Ferrer-Cascales, y Richart-Martínez, 2001) y consideran que los servicios sanitarios son poco útiles para prevenir los problemas de salud que consideran prioritarios (drogas, por ejemplo). En las mujeres, la entrada al mercado laboral se asocia con la adopción de conductas más insalubles, a excepción del sobrepeso (Artazcoz, Moya, Vanaclocha y Pont, 2004). La salud, en definitiva, no es esencialmente la variable de resultado de la intervención médica sino un producto psicoecológico, producto de una interrelación compleja de factores comportamentales, genéticos, ambientales, económicos, políticos y sociales. La salud es, pues, un recurso de oportunidad para la vida cotidiana y no un fin en sí misma. Los problemas de salud son un episodio biográfico más del que interesa, como foco de atención, el impacto de su detección, tratamiento y los determinantes de los resultados de la persona (Kaplan, 1990, 1994, 2003). Desde ahí, también, la consideración de la salud en la enfermedad como un recurso de oportunidad que fortalece el significado de la vida a pesar de encontrarse en un estado de bienestar comprometido (Moch, 1998). Se insiste, por ello, en posibilitar un contrato social entre el paciente y el clínico que permita al paciente establecer sus deseos y expectativas de vida acorde a su propio modo de vida (Barofsky, 2003). La misma adversidad puede ser una vía de ayuda para encontrar nuevos significados en la vida. La adversidad y el sufrimiento psicológico asociado no tienen porqué considerarse como algo anormal, negativo e inapropiado, que hay perentoriamente que eliminar o cambiar (Wilson y Luciano Soriano, 2002). El sufrimiento es consustancial a la vida y su control es un buen marcador de bienestar y calidad de vida.

Una atención sanitaria enfocada a la enfermedad, desprovista de la persona en su contexto, empuja a muchos ciudadanos a la 
búsqueda de otras alternativas de salud. No es tampoco una novedad el fenómeno de la utilización por parte de los ciudadanos de instancias asistenciales y terapéuticas diferentes de las oficialmente reconocidas. La utilización de la autoayuda y la automedicación, o la utilización de "sanadores" situados en contextos diferentes del sistema médico dominante (el concepto conocido como pluralismo asistencial), es un fenómeno que tanto historiadores como antropólogos han venido prestando atención en los últimos dos decenios (Perdiguero, 2004). Como señala este autor, en realidad en todo tiempo y lugar lo que han ofertado los médicos sólo ha sido, y sigue siendo, una fracción de lo que la población utiliza para resolver sus problemas de salud.

Los médicos necesitan ser capaces de informar a los pacientes de las influencias psicológicas en el proceso de enfermedad pero sin sobrecargarles de responsabilidad. Se habla de una "tiranía de la salud" cuando las costumbres sociales y los estilos de vida de las personas se regulan con criterios médicos (Fitzpatrick, 2001). Esta medicalización de la nueva moral convierte al médico en el nuevo sacerdote de la sociedad del bienestar (Gracia, 1983). La virtud y el vicio se han traducido en prescripciones sanitarias sanas e insanas acerca de estilos de vida buenos y malos ligados a la alimentación, al ejercicio físico, al estrés, etcétera. La idea popular de que el estrés causa enfermedades es demasiado simplista e irrealista.

A pesar de los avances en el conocimiento de las conexiones entre los procesos biológicos, psicológicos y sociales de interés para la salud, el enfermar y la enfermedad, no se ha cubierto ni explicado suficientemente su rico y enorme potencial. Los médicos tienen un importante reto que atender desde esta perspectiva: la problemática crónica de salud y las consecuencias de la misma sobre la discapacidad y el estado de salud. Los pacientes crónicos afrontan un buen número de estresores, la gran mayoría de los cuales son de naturaleza negativa y aversiva (Reig, Guerra y Brengelmann, 1983). La persona con problemática crónica de salud debe aprender a convivir con esta condición de salud durante toda su vida sin una perspectiva de curación. El médico debe saber, en este sentido, que el grado y tipo de estrés referido o experimentado no depende exclusivamente del diagnóstico o de la enfermedad per se, sino, en 
gran medida, de las características personales tales como la edad, el género, el nivel de estudios, la situación de vida o la duración de la enfermedad (Heijmans, Rijken, Foets, de Ridder, Schreurs y Bensing, 2004). Los pacientes con diferentes enfermedades crónicas tienen que afrontar una serie de estresores comunes que tienen que ver con el grado de amenaza vital que impone su condición de salud, el deterioro progresivo, el impacto en el grado de controlabilidad personal, y una sintomatología nuclear alrededor de la fatiga y vigor. La naturaleza esencial de esta tarea es la misma para todos ellos. Si esto es asi, cualquier paciente crónico podría beneficiarse de programas específicos que atendieran eficazmente la vicisitudes de esa serie de tareas básicas (Heijmans, Rijken, Foets, de Ridder, Schreurs y Bensing, 2004). Ocurre también que en las experiencias negativas ligadas a la enfermedad crónica algunos pacientes descubren o saben encontrar elementos positivos (cambiar prioridades de vida; dar mayor importancia a las relaciones con la familia y amigos; aceptarse o comprenderse mejor, etc.) que pueden redundar en su propio bienestar y el de su familia, o en otros resultados de calidad de vida y salud. Así, Carver y Antoni (2004) han encontrado que aquellas pacientes con cáncer de mama que referian inicialmente haber encontrado aspectos positivos en su situación neoplásica presentaban al cabo de 5 u 8 años de seguimiento un funcionamiento afectivo y psicológico significativamente mejor en comparación con las que no habían extraído elementos beneficiosos de su situación. Resultados similares han encontrado también Tomich y Helgeson (2004), quienes además añaden que son las mujeres que perciben una enfermedad más grave al inicio del problema las que suelen encontrar y referir más aspectos positivos o beneficiosos.

En este sentido, la persona tiene mucho que decir y hacer. Uno de los puentes de entendimiento más fructíferos, prácticos y demandados entre la medicina y la psicología consiste en el área de la calidad de vida relacionada con la salud. Incorporar la perspectiva de resultados del paciente es una exigencia cada vez mayor en cualquier ensayo clínico y en muchos otros estudios de evaluación de resultados. Cobra cada día más fuerza el modelo de resultados de salud, propuesto por Kaplan (2003), atender adecuadamente las exigencias del nuevo modelo de salud centrado en la persona. Una 
evaluación de los posibles beneficios de una intervención sanitaria sirı proporcionar evidencia del impacto de la misma sobre el paciente, en términos de estado de salud y de calidad de vida, es desde cualquier punto de vista incompleta. El paciente precisa referir cómo valora y experimenta los aspectos ligados a su estado de salud (vigor, dolor, discapacidad) así como a aspectos más ámplios de funcionamiento y bienestar físico, psicológico y social. Desde hace unos cuantos años se comprueba un entusiasmo considerable acerca del potencial de algunas técnicas de medida como los cuestionarios para proporcionar una evidencia precisa y válida de resultados de salud desde la perspectiva del paciente. Garret, Schmidt, Mackintosch y Fitzpatrick (2002) han analizado, con este objetivo, el grado de desarrollo y aplicación de los instrumentos de calidad de vida relacionada con la salud y su disponibilidad para cualquier aspecto de la investigación y práctica clínicas. Estos autores encuentran un crecimiento espectacular del número de referencias de instrumentos (en inglés) a lo largo del periodo estudiado (1990-1999) en las principales bases (AMED, Biological Abstracts, British Nursing Index, Cinahl, Econlit, Embase, Medline, PAIS International, Psyclnfo, Royal College of Nursing database, Sigle, y Sociological Abstracts) notando la saludable disponibilidad de instrumentos. No obstante, estas medidas varían considerablemente según el problema de salud. Cáncer, reumatología y problemática muscoloesquelética acaparan por especialidad médica (de las 30 analizadas) el mayor número de instrumentos disponibles. Se observa escaso desarrollo en la evaluación de la calidad de vida en quemados, cuidados intensivos, traumatología, personas mayores, y ginecología. De los 3921 instrumentos de resultados de salud que cumplían los requisitos de los criterios de inclusión, el $46 \%$ eran medidas espec/ficas de enfermedad o de población, $22 \%$ instrumentos genéricos, $18 \%$ específicos de dimensión, $10 \%$ medidas de utilidad, y un $1 \%$ instrumentos individualizados de CVRS.

Los ciudadanos están desempeñando cada vez más un papel central y activo en el sistema sanitario y pueden hacer mucho para mejorarlo. Se persigue, con ello, la capacitación de las personas para tomar decisiones en relación con su salud y su atención de forma más autónoma y responsable. Resulta de ayuda conceptual 
aquí la teoría de la autodeterminación de la motivación de Ryan y Decci (2000) en la lucha de las personas por mantener ciertas cualidades fundamentales de la experiencia: las personas quieren sentirse eficaces en sus actividades (competencia), sentir que las cosas que hacen las eligen ellos mismos (autonomia) y sentir una sensación de cercanía con los demás (pertenencia). El usuario de la atención sanitaria puede mejorar el sistema sanitario a través de su compromiso en: facilitar al profesional toda la información pertinente acerca de su proceso de salud e impacto sociopersonal; participando en actividades de educación sanitaria; realizando sugerencias y reclamaciones; eligiendo médico, compañía aseguradora, centro sanitario, tratamiento, etc.; participando con el profesional de la salud en la adopción y cumplimiento de la estrategia sanitania idónea; o manifestando su grado de satisfacción tanto del proceso de la atención sanitaria como del resultado de la intervención. La defirición de políticas de salud y servicios precisa obtener información directa de estos ciudadanos y las encuestas de salud son uno de los instrumentos más valiosos para obtenerla (Brugulat, Séculi, Medina, Juncá, Martínez, Martínez, y Treserras, 2003; Gispert, Puig, Puigfàbregas, Tresserras, y Busquets, 2003). Hacemos referencia a la Encuesta de Salud de Cataluña porque hasta la fecha es la única que incorpora una medida de calidad de vida relacionada con la salud con criterios métricos de calidad. De los resultados, se puede destacar que la mayor parte de la población declara gozar de buena salud (el $78 \%$ ); casi un $65 \%$ manifiesta no tener dificultades en las dimensiones de calidad de vida en funcionamiento físico, psicológico y social; prácticamente el $70 \%$ declara que tiene 0 ha presentado trastornos crónicos de salud; $y$ el $12,5 \%$ de la población manifiesta tener alguna discapacidad, es decir, problemas de salud que suponen restricciones funcionales que limitan la capacidad de la persona para desarrollar su actividad de una manera considerada normal. Los autores constatan que, si bien ha aumentado (comparando la encuesta de 1994 frente a la de 2002) la declaración de padecer enfermedades crónicas y una mayor utilización de medicamentos, se observa un incremento de la proporción de personas que hacen una valoración positiva de su estado de salud (Brugulat et al., 2003). 
Con la medición, investigación y gestión de los resultados en salud se quiere valorar, de manera continuada y en relación con los recursos invertidos, las consecuencias de las decisiones e intervenciones sanitarias en términos tanto de la salud individual y poblacional como de la satisfacción de los usuarios. La investigación de resultados en salud consiste en la aplicación de una serie de métodos científicos para la obtención de resultados de en salud de tipo clínico, económico y otros centrados en la persona o usuario. Es, por tanto, un concepto más amplio que el de la evaluación de intervenciones sanitarias, tradicionalmente centradas en los resultados de tipo clinico y en la eficacia de la intervención. la revisión de la bibliografía, las guias de práctica clínica, el ensayo clínico, el meta-análisis, los estudios en calidad de vida, la investigación de la satisfacción, los estudios de efectividad, los estudios observacionales, el uso de recursos sanitarios, la modelización, los estudios epidemiológicos, 0 la evaluación económica contorman las principales áreas de investigación actual para la obtención de resultados en salud en condiciones de práctica clínica habitual (Badía, 2000). Se precisa, por ello, de cuestionarios de resultado centrados en la persona en los que se recojan los objetivos, valores y efectos del tratamiento que el paciente y familia priorizan. Disponemos de datos que nos informan de las cosas que la gente considera más importantes en su vida. Así, por ejemplo, las personas valoran, en orden de prioridad, las relaciones con la familia y parientes, la propia salud, la salud de alguien cercano, las condiciones económicas y domésticas de vida, y las actividades de tiempo libre y vida social. En esta línea, las cinco áreas más frecuentemente referidas como importantes serían las condiciones de vida, las relaciones familiares, la propia salud, la salud de la persona querida, y, por último, la actividad social y de ocio (Bowling, 1995). Este área de la calidad de vida relacionada con la salud es una realidad de gran interés entre la medicina y la psicología.

Otro importante desafío actual a estudiar desde un modelo de salud centrado en la persona y en su contexto tiene que ver con la discapacidad: Los estudios epidemiológicos longitudinales han demostrado que es un buen predictor de numerosos resultados de salud: es el predictor más fuerte de resultados adversos de salud 
después de la edad, incluyendo la mortalidad, el declinar en funcionamiento, el aumento de enfermedades agudas, el aumento de caídas y lesiones, de utilización de servicios sanitarios y de institucionalización (Guralnik, Fried y Salive, 1996). El estudio de la discapacidad tiene un gran interés por su impacto potencial en la atención sanitaria, programas sociales y los costes derivados para las administraciones públicas, los individuos y sus allegados. La finalidad que se persigue con los estudios de discapacidad consiste en contribuir al diseño de estrategias de atención sanitaria de larga duración, de medidas de política sanitaria, y de políticas sociales que hagan posible un aumento del bienestar de las personas en situaciones de desventaja (Puga González y Abellán García, 2004). El concepto de discapacidad se refiere al impacto que las condiciones agudas o crónicas de salud tienen sobre el funcionamiento de sistemas corporales específicos, y de forma general sobre el comportamiento de las personas para actuar de la forma necesaria, usual, esperada o personalmente deseada, en su medio sociocultural. El modelo de discapacidad que guía la investigación científica en la actualidad es el denominado "proceso de discapacitación" (disablement process) (Verbrugge y Jette, 1994). El proceso de discapacitación propone como vía principal hacia la discapacidad la siguiente secuencia: (1) enfermedad, (2) deficiencia, (3) disfunción, y (4) discapacidad. Valorando las limitaciones en alguna actividad básica o instrumental en su vida, o en sus funciones cognitivas, que impiden el normal desarrollo de la vida cotidiana, Puga González y Abellán Garcia, 2004) estiman una prevalencia de discapacidad del $6,8 \%$ de la población, a partir de la Encuesta sobre discapacidades, deficiencias y estado de salud (EDDES) realizada en el año 1999.

El profesional de la salud debe estar atento a los modelos tácitos de discapacidad que subyacen a muchos de los instrumentos de calidad de vida y que se pueden sintetizar en los siguientes cuatro modelos: el funcional ("soy incapaz de andar"), el comparativo ("estoy tan sano como cualquiera que concozco"), el de malestar subjetivo ("tengo problemas para subir y bajar escaleras") y el de dependencia ("sólo puedo vestirme con la ayuda de alguien") (Ziebland, Fitzpatrick y Jenkinson, 1993). Hay que estar atentos, además, a las paradojas que se producen y que pueden retratar una 
situación en la que tener un problema crónico grave mejora la calidad de vida. Un buen número de pacientes con cáncer refiere beneficios derivados de su enfermedad, tales como un aumento de la capacidad de apreciar cada día, sentimientos de fuerza personal, una mayor compasión de cara a los demás, una mejor comprensión de las cosas, o una mayor seguridad personal. Equiparando calidad de vida con normalidad en funcionamiento físico se ignora el amplio abanico de posibilidades con los que la gente se adapta satisfactoriamente a la vida a pesar de importantes limitaciones fisicas (Koch, 2000). La paradoja de la discapacidad acentúa la importancia de la experiencia personal con la discapacidad en la defirición del sí mismo (autoconcepto) de la persona, y su mirada del mundo, del contexto social y de las relaciones interpersonales. Albrecht y Devlieger (1999) argumentan que una buena calidad de vida, a pesar de las condiciones adversas, refleja un balance reconstruido entre cuerpo, mente y espíritu. En su estudio, estos autores encuentran que la gente con discapacidad notoria que refiere una calidad de vida excelente 0 buena (un 54\%), expresa su bienestar en términos de conocimiento de su deficiencia, encontrarse con control de cuerpo y mente, ser capaz de llevar a cabo los roles esperados, tener una perspectiva en la vida de "puedo hacer", encontrar un propósito, significado y armonía en la vida, tener una mirada espiritual, o sentirse satisfecho en comparación con otros en situaciones similares. Aquellos (un $45 \%$ ) que refieren una mala o regular calidad de vida manifiestan como nuclear de ello la presencia de dolor, fatiga, pérdida de control de las funciones corporales, o no tener un sentido de la vida claro o una mirada espiritual de la misma (Albrecht y Devlieger, 1999).

Otro aspecto a resaltar es que cualquier profesional de la salud tiene que tener presente que toda planificación social y sanitaria debe realizarse tomando en consideración que el cuidado de la salud se realiza sobre todo desde las familias, y que han sido tradicionalmente las mujeres las encargadas de prestar los servicios de cuidado (Durán, 2004; García-Calvente, Mateo-Rodriguez y Eguiguren, 2004). Los hogares son las unidades sociales que mediatizan la relación entre los individuos y el sistema sanitario. La ganancia observada en los indicadores de esperanza de vida, esperanza de vida libre de incapacidad y esperanza de vida en buena salud (Gisbert 
et al., 2003), la progresiva incorporación de las mujeres al empleo remunerado, el surgimiento de nuevas formas de estructura familiar, las crecientes aspiraciones de calidad de vida, y la llegada de gran número de inmigrantes, están modificando las condiciones en que se producen las demandas de atención sanitaria (Durán, 2004; Jansà y García de Olalla, 2004).

Por último, en este modelo de salud centrado en la persona y su contexto tiene un especial interés la atención a la salud cuando se trata de enfrentarse al final de la vida. Sanz Ortiz (2004) recomienda que se deje de considerar la medicina como algo que consiste en que la gente no se muera, y se incorpore definitivamente la muerte a la vida. Concluye, además, en la necesidad de permitir a aquellos enfermos moribundos, que "padecen" la vida, una sedación terminal que procure hacer la última etapa del viaje de la vida de forma humanizada; encontrar el límite del esfuerzo terapéutico, de manera que se evite la futilidad o ensañamientos terapéuticos; un acompañamiento basado en la afectividad y compañía que procure una muerte digna; y el dominio de las modernas técnicas en medicina paliativa tendentes a que también el último acto de la vida lo protagonice la propia persona en un contexto de respeto de su proyecto de vida, prioridades y valores. Sin duda, la aplicación de los estándares de formación psicológica en cuidados paliativos (Fernández Fernández et al., 2004) es una buena propuesta para un modelo de salud centrado en la persona y su contexto.

\section{BIBLIOGRAFÍA}

Albrecht, G.L., \& Devlleger, P.J. (1999). The disability paradox: high quality of life against all odds. Social Science and Medicine, 48: 977-988.

Alcaraz, J. (2005). [Entrevista al doctor Ciril Rozman] "Hi ha massa especialistes sense coneixements bàsics". El Temps, de l'1 al 7 de febrer de 2005: 50-52.

Álvarez Peláez, R. (2004). Una mirada sobre 《<lo biológico » en la psiquiatria española. Frenia, IV: 7-30.

Anderson, B.L. (2002). Biobehavioral outcomes following psychological interventions for cancer patients. Joumal of Consulting and Clinical Psychology, 70. 590-610. 
Anónimo (2004). Editorial. El paciente en España, Jano, LXVI: 1516.

Aranaz, J.M., Albar, C., Gea, M.T., y León, M.T. (2004). Efectos adversos en la asistencia hospitalaria. Una revisión crítica. Medicina Clínica (Barcelona), 123. 21-25.

Artacoz, L., Moya, C., Vanaclocha, H., y Pont, P. (2004). La salud de las personas adultas. Gaceta Sanitaria, 18 (Supl. 1): 56-68.

Ashton, J. (1987). La promoción de la salud, un nuevo concepto para una nueva sanidad. Las ciudades sanas, una iniciativa de la nueva sanidad. Valencia, Generalitat Valenciana, Conselleria de Sanitat i Consum, IVESP. Badía, X. (Ed.) (2000). La investigación de resultados en salud. De la evidencia a la práctica clínica. Barcelona, Edimac.

Barlow, D.H. (2004). Psychological treatments. American Psychologist, 59. 869-878.

Barofsky, I. (2003). Patients' rights, quality of life, and health care system performance. Quality of Life Research, 12. 473-484.

Blumenthal, J., Sherwood, A., Gullette, E., Georgiades, A., \& Tweedy, D. (2002). Biobehavioral approaches to the treatment of essential hypertension. Joumal of Consulting and Clinical Psychology, 70. 569589.

B.O.E. (2003a). Real Decreto 1277/2003, de 10 de octubre, por el que se establecen las bases generales sobre autorización, servicios y establecimientos sanitarios. Número 254, jueves 23 octubre 2003: 37893-37902.

B.O.E. (2003b). Ley 44/2003, de 21 de noviembre, de ordenación de las profesiones sanitarias. Número 280, sábado 22 noviembre 2003: 4144241458.

Bonanno, G.A. (2004). Loss, trauma, and human resilience. Have we underestimated the human capacity to thrive after extremely aversive events? American Psychologist, 59. 20-28.

Bowling, (1995). What things are important in people's lives? A survey of the public's judgements to inform scales of health related quality of life. Social Science and Medicine, 10. 1447-1462.

Bray, J.H. (2004). Training primary care psychologists. Joumal of Clinical Psychology in Medical Settings, 11: 101-107.

Brugulat, P., Sécull, E., Medina, A., Juncá, S., Martínez, V., Martínez, D., y Treserras, R. (2003). Encuesta de Salud de Cataluña. Percepción de la salud, utilización y satisfacción con los servicios asistenciales desde la perspectiva de los ciudadanos. Medicina Clínica (Barcelona), 121 (Supl. 1): $122-127$.

Buela-Casal, G., Gil Roales-Nieto, J., Slerra, J.C., Bermúdez, M.P., Agudelo, D., Breton, J., y Teva, I. (2005). La imagen de la psicología como profesión sanitaria en profesores de psicología y de medicina. Papeles del Psicologo, (en prensa). 
Cabrero García, J. y RIchart Martínez, M. (2001). Investigar en enfermería. Concepto $y$ estado actual de la investigación en enfermería. Alicante, Publicaciones Universidad de Alicante.

Carver, C.S., \& AntonI, M.H. (2004). Finding benefit in breast cancer during the year after diagnosis predicts better adjustment 5 to 8 years after diagnosis. Health Psychology, 23: 595-598.

Cohen, S. (2004). Social relationships and health. American Psychologist, November. 676-684.

Davo, M.C., y Álvarez-Dardet, C. (2003). El genoma y sus metáforas. ¿Detectives, héroes o profetas? Gaceta Sanitaria, 17: 59-65.

Delgado, A., y López-Fernández, L.A. (2004). Práctica profesional y género en atención primaria. Gaceta Sanitaria, 18 (Supl. 1): 112-117.

Demarbre Zboril, V. (2001). Health psychology in Catalonia in 2000: Current view and future perspective. Joumal of Clinical Psychology in Medical Settings, 8: 21-25.

Dubos, R. (1978). Health and creative adaptation. Human Nature, 1: 7482.

Durán, M.A. (2004). Las demandas sanitarias de las familias. Gaceta Sanitania, 18 (Supl. 1): 195-200.

Engel, G. (1977). The need for a new medical model: A challenge for biomedicine. Science, 196. 129-136.

Fernández Fernández, M.E., Agulrán Clemente, P., Amurrlo López de Gastlain, L.M., Limonero Garcia, J.T., Martínez garcia, M., y Urbano Salgado, S. (Grupo de trabajo de formación de psicólogos, SECPAL) (2004). Estándares de formación psicológica en Cuidados Paliativos. Medicina Paliativa, 11: 174-179.

Ferreres, J., Baños, J.E., y Farré, M. (2004). Efecto nocebo: la otra cara del placebo. Medicina Clínica (Barcelona), 122. 511-516.

Fitzpatrick, M. (2001). The tiranny of health. Doctors and the regulation of lifestyle. London, Routledge.

Garcia-Calvente, M., Mateo-Rodríguez, I., y Egulguren, A.P. (2004). El sistema informal de cuidados en clave de desigualdad. Gaceta Sanitaria, 18 (Supl. 1): 132-139.

García-Campayo, J., y Sanz Carrllio, C. (2002). Salud mental en inmigrantes: el nuevo desafio. Medicina Clínica (Barcelona), 118: 187-191.

Garrat, A., Schmidt, L., Mackintosh, A., \& Fitzpatrick, R. (2002). Quality of life measurement: bibliographic study of patient assessed health outcome measures. British Medical Joumal, 324: 1-5.

Gatchel, R.J. (2004). Comorbidity of chronic pain and mental health disorders: The biopsychosocial perspective. American Psychologist, November. 795805. 
Gil Roales-Nieto, J. (2004). Psicología de la salud. Aproximación histórica, conceptual y aplicaciones. Madrid, Pirámide.

Gispert, R., Puig, X., Puigfàbregas, A., Tresserras, R., y Busquets, E. (2003). Esperanza de vida libre de incapacidad y esperanza de vida en buena salud en Cataluña 1994-2000. Medicina Clínica (Barcelona), 121 (Supl. 1): 128-132.

Gracla, D. (1983). ¿Profesión o sacerdocio? Jano, $n^{9}$ extraordinario: 38-52. Guralnik, J.M., Frled, L.P., \& Salive, M.E. (1996). Disability as a public health outcome in the aging population. Annual Review of Public Health, 17: $25-46$.

Heljmans, M., Rljken, M., Foets, M., de Ridder, D., Schreurs, K., \& Bensing, J. (2004). The stress of being chronically ill: From diseasespecific to task-specific aspects. Journal of Behavioral Medicine, 27: 255271.

Hernán, M., Fernández, A., y Ramos, M. (2004). La salud de los jóvenes. Gaceta Sanitaria, 18 (Supl. 1): 47-55.

Hodges, L.J., Humphrls, G.M., \& Macfariane, G. (2005). A meta-analytic investigation of the relationship between the psychological distress of cancer patients and their carers. Social Science \& Medicine, 60. 1-12.

Hoiman, H. (2004). Chronic disease - The need for a new clinical education. JAMA, 292, 1057-1059.

Hong, B.A., \& Leventhai, G. (2004). Partnerships with psychiatry and other clinical disciplines: A key to psychology's success in U.S. Medical Schools. Joumal of Clinical Psychology in Medical Settings, 11: 135-140.

Jansà, J.M. y García de Olalia, P. (2004). Salud e inmigración: realidades y nuevos retos. Gaceta Sanitaria, 18 (Supl. 1): 207-213.

Jiménez Jiménez, C. (2001). Ciencias psicosociales aplicadas a la salud II. Aportaciones a los profesionales de la salud desde la antropología, la sociología y la psicología. Córdoba, Senvicio Publicaciones Universidad de Córdoba.

Johnson, S.B. (2004). Integrating behavior into health research and health care: One psychologist's journey. Joumal of Clinical Psychology in Medical Settings, 11: 91-99.

Jovell, A.J. (1999). Medicina basada en la afectividad. Medicina Clínica (Barcelona), 113: 173-175.

Kaplan, R. (1990). Behavior as the central outcome in health care. American Psychologist, 45: 1211-1220.

Kaplan, R. (1994). The Ziggy Theorem: Toward an outcomes-focused health psychology. Health Psychology, 13: 451-460.

Kaplan, R. (2003). The significance of quality of life in health care. Quality of Life Research, 12 (Suppl. 1): 3-16. 
Kaplan, R., \& GroessI, E. (2002). Applications of cost-effectiveness: Methodologies in behavioral medicine. Joumal of Consulting and Clinical Psychology, 70. 482-493.

Klecolt-Glaser, J., \& Glaser, R. (2002). Psychoneuroimmunology: Psychological influences on immune function and health. Joumal of Consulting and Clinical Psychology, 70. 537-547.

King, C.A. (2004). Psychologists in academic health settings: Key contributors to dynamic interplay among research, clinical practice, and policy domains. Joumal of Clinical Psychology in Medical Settings, 11: 83-90.

Knoops, K.T., de Groot, L.C., Kromhout, D., Perrln, A.E., MorelrasVarela, O., Menottl, A., \& van Staveren, W.A. (2004). Mediterranean diet, lifestyle factors, and 10-year mortality in elderly european men and women. The HALE Project. JAMA, 292. 1433-1439.

Koch, T. (2000). Life quality vs the 'quality of life': assumptions underlying prospective quality of life instruments in health care planning. Social Science \& Medicine, 51: 419-427.

Kubzansky, L.D., Sparrow, D., Vokonas, P., \& Kawachl, I. (2001). Is the glass half empty or half full? A prospective study of optimism and coronary heart disease in the Normative Aging Study. Psychosomatic Medicine, 63: $910-916$.

Kush, F.R. (2001). Primary care and clinical psychology: Assessment strategies in medical settings. Journal of Clinical Psychology in Medical Settings, 8: 219-228.

Laín Entralgo, P. (1983). La relación médico-enfermo. Madrid, Alianza Editoriai.

León Rubio, J.M., Medina Anzano, S., Barriga Jiménez, S., Ballesteros Regaña, A., y Herrera Sánchez, I. (2004). Psicologla de la salud y de la calidad de vida. Barcelona, Editorial UOC.

Leventhal, H., Leventhal, E., \& Cameron, L. (2001). Representations, procedures and affect in illness self-regulation: A perceptual-cognitive model. En: A. Baum, T. Revenson, \& J.E. Singer (Eds.). Handbook of health psychology. Mahwah, NJ, Erlbaum: 19-28.

Levy, B.R., Slade, M.D., Kunkel, S.R., \& Kasl, S.V. (2002). Longevity increased by positive sel-perceptions of aging. Joumal of Personality and Social Psychology, 83: 261-270.

Lizán, L. (2004). Medicina centrada en las personas. Jano, LXVII: 144.

Lockwood, S. (2004). "Evidence of me" in evidence based medicine? British Medical Journal, 329: 1033-1035.

Marañón Cabelio, A. (2004). La medicina actual: luces y sombras. Medicina Clínica (Barcelona), 122, 623. 
Marsh, D.R., Schroeder, D.G., Dearden, K.A., Sternin, J., \& Sternin, M. (2004). The power of positive deviance. British Medical Journal, 329. 1177-1179.

Martire, L.M., Lustig, A.P., Schulz, R., Miller, G.E., \& Helgeson, V.S. (2004). Is it beneficial to involve a family member? A meta-analysis of psychosocial interventions of chronic illness. Health Psychology, 23: 599611.

Maruta, T., Colligan, R.C., Malinchoc, M., \& Offord, K.P. (2000), Optimists vs. pessimists: Survival rate among medical patients over a 30-year period. Mayo Clinic Proceedings, 75: 140-143.

Mckeown, T. (1976). The role of medicine: dream, mirage or nemesis? Nuffield Provincial Hospitals Trust.

Melamed, B.G. (1995). The neglected psychological-physical interface. Health Psychology, 14: 371-373.

Moch, S.D. (1998). Health-within-illness: concept development through research and practice. Joumal of Advanced Nursing, 28: 305-310.

Moore, P.J., Slckel, A.E., Malat, J., WIIllams, D.R., Jackson, J.S., \& Adler, N.E. (2004). Psychosocial factors in medical and psychological treatment avoidance: the role of the doctor-patient relationship. Joumal of Health Psychology, 9: 421-433.

Nieto-Munuera, J., Abad Mateo, M.A., Esteban Albert, M., y Tejerina Arreal, M. (2004). Psicología para ciencias de la salud: estudio del comportamiento humano ante la enfermedad. Madrid, McGraw-Hill/ Interamericana.

Nitzan, U., \& Llchtenberg, P. (2004). Questionnaire survey on use of placebo. British Medical Journal, 329: 944-946.

Nlura, R., \& Abrams, D. (2002). Smoking cessation: Progress, priorities and prospectus. Journal of Consulting and Clinical Psychology, 70. 510-525.

Novoa Jurado, A.J., Molina Durán, F., y Luna Maldonado, A. (2004). Reconstrucción del pensamiento médico: fundamentos del pragmatismo clínico. Medicina Clínica (Barcelona), 123. 345-351.

O'Donohue, W., C Cuccuare, M.A. (2005). The role of psychological factors in medical presentations. Joumal of Clinical Psychology in Medical Settings, 12. 13-24.

Pelechano, V. (1996). Psicología clínica, psicología de la salud y la búsqueda del santo Grial. Análisis y Modificación de Conducta, 22. 323-363.

Pelechano, V. (1996). Salud, enfermedad, clínica y psicología: un laberinto con una salida sensata y muchos caminos ciegos. Análisis y Modificación de Conducta, 22. 365-382.

Perdiguero, E. (2004). El fenómeno del pluralismo asistencial: una realidad por investigar. Gaceta Sanitaria, 18 (Supl. 1): 140-145. 
Pérez Álvarez, M. (1990). Médicos, pacientes y placebos. El factor psicológico en la curación. Oviedo, Pentalfa Ediciones.

Pérez Álvarez, M., Fernández Hermida, J.R., Fernández Rodríguez, C., y Amigo Vázquez, I. (2003). Gula de tratamientos psicológicos eficaces. l: Adultos. II: Psicologia de la salud. III: Infancia y adolescencia. Madrid, Pirámide.

Pico della Mirandola, G. (1988). Discurso sobre la dignidad del hombre. Traducción, Introducción, Edición y notas de Pedro J. Quetglas. Barcelona, PPU.

Puga González, M. D. y Abellán García, A. (2004). El proceso de la discapacidad. Un análisis de la Encuesta sobre discapacidades, deficiencias y estado de salud. Alcobendas (Madrid), Fundación Pfizer.

Ray, O. (2004). How the mind hurts and heals the body. American Psychologist, 59. 29-40.

Reig-Ferrer, A., Guerra, J. und Brengelmann, C.J. (1983). Die dimensionale Analyse des Stressverhaltens. En: J. C. Brengelmann und G. Bühringer (Hrsg.): Therapieforschung für die Praxis 3. München, Gerhard Röttger Verlag: 261-276.

Reig-Ferrer, A., Cabrero-García, J., Ferrer-Cascales, R., y RichartMartínez, M. (2001). La calidad de vida y el estado de salud de los estudiantes universitarios. Alicante, Universidad de Alicante.

Roter, D.L., \& Hall, J.A. (2004). Physician gender and patient-centered communication: a critical review of empirical research. Annual Roview of Public Health, 25: 497-519.

Rozensky, R.H., Johnson, N., Goodheart, C., \& Hammond, R. (2003). Psychology builds a healthy world: Research and practice opportunities. Washington, DC, American Psychological Association.

Ryan, R.M., \& Deci, E.L. (2000). Self-determination theory and the facilitation of intrinsic motivation, social development, and well-being. American Psychologist, 55: 68-78.

Saab, P.G., McCalla, J.R., Coons, H.L., Christensen, A.J., Kaplan, R., Johnson, S.B., Ackerman, M.D., Stepanski, E., Krantz, D.S., \& Melamed, B. (2004). Technological and medical advances: implications for health psychology. Health Psychology, 23: 142-146.

Sanz Ortiz, J. (2002). ¿Qué es bueno para el enfermo? Medicina Clínica (Barcelona), 119. 18-22.

Sanz Ortiz, J. (2004). La sedación en el final de la vida. Modicina Clínica (Barcelona), 123: 423-425.

Seguí Díaz, M., Linares Pou, L., Blanco López, W., Ramos Alelxades, J., y Torrent Quetglas, M. (2004). Tiempos durante la visita médica en atención primaria. Atención Primaria, 33. 496-502. 
Sierra, J.C., Carrasco, T.J., \& Buela-Casal, G. (1995). Daytime sequelae of placebo use of a hypnotic in healthy volunteers. Medical Science Research, 23. 683-684.

Sierra, J.C., Luna-Villegas, G., Buela-Casal, G., \& Fernández-Guardiola, G. (1997). The assessment of residual effects of a single dose of diazepam on visually-defined EEG pattems. Joumal of Psychopharmacology, 11: 371-376.

Smlth, T.W., Kendall, P., \& Keefe, F. (Eds.) (2002). Behavioral medicine and clinical health psychology [Special issue]. Joumal of Consulting and Clinical Psychology, 70: 459-856.

Smith, T. W. \& Rulz, J.M. (2002). Psychosocial influences on the development and course of coronary heart disease: Current status and implications for research and practice. Joumal of Consulting and Clinical Psychology, 70. 548-568.

Suls, J. \& Rothman, A. (2004). Evolution of the Biopsychosocial Model: Prospects and Challenges for Health Psychology. Health Psychology, 23. 119-125.

Taylor, S.E., Kemeny, M.E., Reed, G.M., Bower, J.E., \& Gruenewald, T.L. (2000). Psychological resources, positive illusions, and health. American Psychologist, 55: 99-109.

Teresa de Jesús (1577/1940). Obras completas. Madrid, M. Aguilar Editor.

Tomich, P.L., \& Helgeson, V.S. (2004). Is finding something good in the bad always good? Benefit finding among women with breast cancer. Health Psychology, 23. 16-23.

Tovlan, S.M., Rozensky, R.H., \& Sweet, J.J. (2003). A decade of clinical psychology in medical settings: The short longer view. Joumal of Clinical Psychology in Medical Settings, 10. 1-8.

Turabián Fernández, J.L. y Pérez Franco, B. (2004). Algunos problemas educativos prioritarios a resolver en la formación del médico de familia. Atención Primaria, 33. 507-509.

Verbrugge, L.M., \& Jette, A.M. (1994). The disablement process. Social Science and Medicine, 38. 1-14.

Wade, D.T., \& Halligan, P.W. (2004). Do biomedical models of illness make for good healthcare systems? British Medical Joumal, 329. 1398-1401.

Wilson, K.G. y Luclano, M.C. (2002). Terapia de Aceptación y Compromiso $(A C T)$. Un tratamiento conductual orientado a valores. Madrid, Pirámide.

White, K. (1991). Healing the schism. Epidemiology, medicine and the public's health. New York, Springer Verlag.

Ziebland, S., Fitzpatrick, R., \& JenkInson, C. (1993). Tacit models of disability underlying health status instruments. Social Science and Medicine, 37: 69-75. 